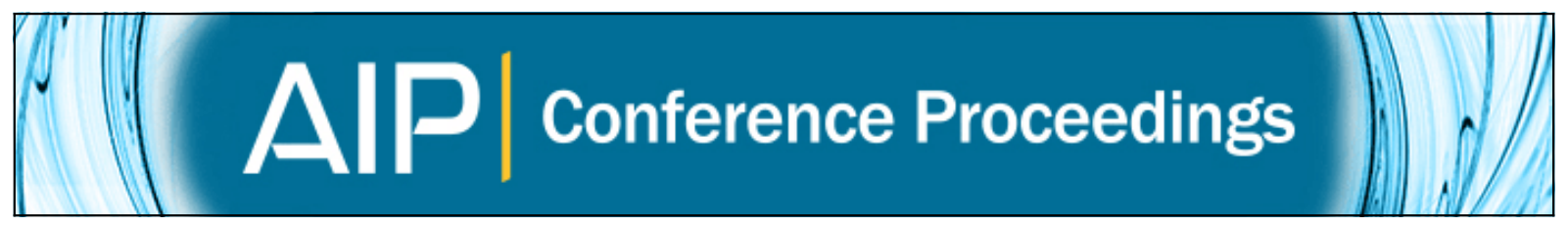

\title{
Plastic Instability in Complex Strain Paths Predicted by Advanced Constitutive Equations
}

Marilena C. Butuc, Frédéric Barlat, José J. Gracio, and Gabriela Vincze

Citation: AIP Conference Proceedings 1383, 194 (2011); doi: 10.1063/1.3623611

View online: http://dx.doi.org/10.1063/1.3623611

View Table of Contents: http://scitation.aip.org/content/aip/proceeding/aipcp/1383?ver=pdfcov

Published by the AIP Publishing

\section{Articles you may be interested in}

Advances of Plasticity Experiments on Metal Sheets and Tubes and Their Applications to Constitutive Modeling AIP Conf. Proc. 778, 20 (2005); 10.1063/1.2011190

Thermo-elasto-visco-plastic constitutive equations fully coupled with ductile damage. Application to metal cutting by chip formation

AIP Conf. Proc. 712, 1365 (2004); 10.1063/1.1766719

Constitutive models for PBX at high strain rate

AIP Conf. Proc. 505, 687 (2000); 10.1063/1.1303566

Problems in Modeling Viscoelastic Flows with Integral Constitutive Equations: Constitutive Predictions and Numerical Instability

J. Rheol. 33, 1047 (1989); 10.1122/1.550045

Plasticity Symposium at New Haven

J. Rheol. 3, 373 (1932); 10.1122/1.2116500 


\title{
Plastic Instability in Complex Strain Paths Predicted by Advanced Constitutive Equations
}

\author{
Marilena.C. Butuc ${ }^{\mathrm{a}}$, Frédéric Barlat ${ }^{\mathrm{a}, \mathrm{b}}$, José J. Gracio ${ }^{\mathrm{a}}$ and Gabriela Vincze ${ }^{\mathrm{a}}$ \\ ${ }^{a}$ Centre for Mechanical Technology and Automation, University of Aveiro, Campus Universitário de Santiago, \\ 3810-193 Aveiro, Portugal \\ ${ }^{b}$ Graduate Institute of Ferrous Technology, Pohang University of Science and Technology, San 31 Hyoja-dong, \\ Nam-gu, Pohang, Gyeongbuk 790-784, Republic of Korea
}

\begin{abstract}
The present paper aims at predicting plastic instabilities under complex loading histories using an advanced sheet metal forming limit model. The onset of localized necking is computed using the Marciniak-Kuczinsky (MK) analysis [1] with a physically-based hardening model and the phenomenological anisotropic yield criterion Yld2000-2d [2]. The hardening model accounts for anisotropic work-hardening induced by the microstructural evolution at large strains, which was proposed by Teodosiu and $\mathrm{Hu}$ [3]. Simulations are carried out for linear and complex strain paths. Experimentally, two deep-drawing quality sheet metals are selected: a bake-hardening steel (BH) and a DC06 steel sheet. The validity of the model is assessed by comparing the predicted and experimental forming limits. The remarkable accuracy of the developed software to predict the forming limits under linear and non-linear strain path is obviously due to the performance of the advanced constitutive equations to describe with great detail the material behavior. The effect of strain-induced anisotropy on formability evolution under strain path changes, as predicted by the microstructural hardening model, is particularly well captured by the model.
\end{abstract}

Keywords: Forming limits; Constitutive equations; Complex loading; Anisotropic; Hardening; Kinematic PACS: $07.05 . \mathrm{Tp}, 75.47 . \mathrm{Np}, 62.20 . \mathrm{fq}, 81.20 . \mathrm{Hy}, 81.40 . \mathrm{Cd}, 81.40 . \mathrm{Ef}, 83.10 . \mathrm{Bb}, 81.40 . \mathrm{Np}$

\section{INTRODUCTION}

The forming limit diagram (FLD) is a constructive concept for characterizing the formability of sheet metal. It was proved to be an essential tool for material selection, design and try out of the tools for deep drawing operations. The maximum admissible limiting strains strongly depend on the deformation mode, loading history and material behavior. To date, the MK method has become one of the most important tools in predicting the sheet metal formability. The selected constitutive equations keep a crucial significance on the prediction of plastic flow localization. For larger strains and for abrupt strain path changes, the evolution of the strain hardening is an issue and is the subject of an intensive recent research. Sheet metal forming processes often impose very intense forming sequences, leading to severe strain-path changes, which drastically influence the forming limits. Therefore, it was introduced the stress-based forming limit concept (FLSD), apparently independent on the strain path changes. Butuc et al.[4] showed that the independence of stress-based forming limits on strain path changes is basically originated on the plastically calculation of the stress state by assuming an isotropic hardening model, no depended on strain path. In addition, Yoshida et al. [5] showed that the work-hardening behavior after prestrain plays an important role in the path-dependence of the FLSD.

The aim of the present work is a more relevant study on the prediction of plastic instabilities under complex loading histories using an advanced sheet metal forming limit model that take into account the strain path induced anisotropy. A particular attention is paid to the origin of the evolution of formability under strain-path changes involving biaxial prestrain followed by uniaxial tension and the reverse sequence. 


\section{THEORETICAL COMPUTATION OF FORMING LIMIT DIAGRAMS}

The simulation of plastic instability is carried out by using a MK-type analysis. This section summarizes the main steps of the theoretical computation. The model is based on the growth by plastic deformation within a thin sheet of an initial defect in the form of a groove-like, narrow band of diminished thickness. A plane stress condition is assumed throughout. The initial inclination angle of the band with respect to the minor principal axis of the stress tensor is denoted by $\psi_{0}$. The initial value of the geometrical defect is characterized by the ratio $\mathrm{e}_{0}^{b} / \mathrm{e}_{0}^{a}$ where $\mathrm{e}_{0}^{a}$ and

$\mathrm{e}_{0}^{b}$ are the initial thickness in the homogeneous region and in the groove respectively. The $x, y, z$-axes correspond to rolling, transverse and normal directions of the sheet, whereas 1 and 2 represent the principal stress and strain directions in the homogeneous region. The set of axes bound to the groove is represented by $n, t, z$, where $t$ denotes the longitudinal axis. It is supposed that the material has a rigid-plastic, rate-independent behaviour. Several isotropic and anisotropic hardening models are considered to describe the hardening behaviour of material. The initial shape of the yield locus is given by the Yld2000-2d plane stress yield function [2]. Small increments of equivalent strain are imposed in the homogeneous region, the corresponding stress and strain states being computed according to the selected constitutive equations. In order to define the strain and stress states within the band, the Newton-Raphson method is applied for solving a system of two polynomial equations resulting from the yield criterion and the requirement of deformation compatibility in the longitudinal direction of the necking band. The Jacobian matrix required in the Newton-Raphson computation is based on an analytical differentiation of the constitutive equations of the model. When the effective strain increment in the band $\left(d \bar{\varepsilon}^{b}\right)$ becomes 10 times larger than the one in the homogeneous zone $\left(d \bar{\varepsilon}^{a}\right)$, it is considered that a plastic flow localization occurs in the band, whereas the corresponding accumulated principal strains in the homogeneous zone define the forming strain limits. For each considered strain path, the analysis is repeated for different values of $\psi_{0}$ (between $0^{\circ}$ and $90^{\circ}$ ) and the limit point on the FLD is obtained through the minimization of the principal strain $\varepsilon_{11}^{a}$ in the homogeneous zone versus $\psi_{0}$. The simulation of the complex strain paths involves a prestrain of the homogeneous zone followed by a more or less sharp change in strain path. Specifically, the sheet metal is unloaded to a zero-stress state after the prestrain and subsequently reloaded following the second strain path. To account for complex strain paths at large plastic strains, the prestrain will be accounted for when considering the evolution of the internal variables of the microstructural hardening model. More precisely, the initial values of the internal variables at the beginning of the second loading are taken equal to their values at the end of the first loading. In addition, it is also considered that the initial band orientation at the beginning of the second strain path is the one achieved at the end of the first deformation stage.

\section{REVIEW OF THE SELECTED CONSTITUTIVE EQUATIONS}

\section{Microstructural Hardening Model}

The microstructural hardening model of Teodosiu and $\mathrm{Hu}$ describes the hardening of the material by four internal state variables, denoted by $\{R, \boldsymbol{X}, \boldsymbol{S}, \boldsymbol{P}\}$. The evolution laws of the internal state variables are written in a workhardening/recovery format, reflecting the dynamic processes of the production/annihilation of dislocations and of the formation/dissolution of dislocation structures.

The evolution of $R$ is given by

$\dot{R}=C_{\mathrm{R}}\left(R_{\text {sat }}-R\right) \dot{\bar{\varepsilon}}, R(0)=0$,

with $C_{\mathrm{R}}$-the rate of the isotropic hardening, $R_{\text {sat }}$-the saturation value of $R$.

The evolution of the tensor $\boldsymbol{P}$, which describes the polarity of planar dislocation structures, is given by

$\dot{\boldsymbol{P}}=C_{\mathrm{P}}(\boldsymbol{A}-\boldsymbol{P}) \dot{\bar{\varepsilon}}, \boldsymbol{P}(0)=0$,

where $\boldsymbol{A}$ is the current direction of the plastic strain rate tensor.

The evolution of the back stress $\boldsymbol{X}$ is governed by the equation

$\dot{\boldsymbol{X}}=C_{\mathrm{X}}\left(\frac{X_{\mathrm{sat}}}{\bar{\sigma}_{e}}\left(\boldsymbol{\sigma}^{\prime}-\boldsymbol{X}\right)-\boldsymbol{X}\right) \dot{\bar{\varepsilon}}, \boldsymbol{X}(0)=0$, 
with $C_{\mathrm{X}}$-the saturation rate of $\boldsymbol{X}, \boldsymbol{X}_{\text {sat }}$-the saturation value of the norm of $\boldsymbol{X}, \boldsymbol{\sigma}^{\prime}$-the deviator stress tensor and $\bar{\sigma}_{e}$ the equivalent stress corresponding to the effective stress tensor $\boldsymbol{\sigma}^{\prime}-\boldsymbol{X} . \boldsymbol{X}_{\text {sat }}$ is assumed as:

$X_{\mathrm{sat}}=X_{0}+(1-m) \sqrt{S_{\mathrm{D}}^{2}+q\left|\boldsymbol{S}_{\mathrm{L}}\right|^{2}}$,

where $X_{0}$ is the initial value of $X_{\text {sat }}$, while $q$ and $m$ are material parameters.

The tensor variable $S$ describing the directional strength of planar dislocation structures is decomposed as

$\boldsymbol{S}=S_{\mathrm{D}} \boldsymbol{A} \otimes \boldsymbol{A}+\boldsymbol{S}_{\mathrm{L}}$

with $S_{\mathrm{D}}=\boldsymbol{A}: \boldsymbol{S}: \boldsymbol{A}$ and $S_{\mathrm{L}}=\left|\boldsymbol{S}_{\mathrm{L}}\right|$.

$S_{\mathrm{D}}$ is related to the currently active slip systems and $\boldsymbol{S}_{\mathrm{L}}$ to the latent part of the dislocation structures. The evolution of $S_{\mathrm{D}}$ is governed by the equation:

$\dot{S}_{\mathrm{D}}=C_{\mathrm{SD}}\left[h_{\mathrm{P}}\left(S_{\mathrm{sat}}-S_{\mathrm{D}}\right)-h_{\mathrm{X}} S_{\mathrm{D}}\right] \dot{\bar{\varepsilon}}$,

where $C_{\mathrm{SD}}$ and $S_{\text {sat }}$ are material parameters. $h_{\mathrm{X}}$ and $h_{\mathrm{P}}$ are 2 functions expressed as:

$h_{\boldsymbol{X}}=\frac{1}{2}\left(1-\frac{\boldsymbol{X}: \boldsymbol{A}}{X_{\mathrm{sat}}\left(\boldsymbol{\sigma}^{\prime}-\boldsymbol{X}\right): \boldsymbol{A}} \bar{\sigma}_{e}\right)$.

$\mathrm{h}_{P}=\left\{\begin{array}{c}1-\frac{\mathrm{C}_{P}}{\mathrm{C}_{S D}+\mathrm{C}_{P}}\left|\frac{\mathrm{S}_{D}}{\mathrm{~S}_{\text {sat }}}-\boldsymbol{P}: \boldsymbol{A}\right| \quad \text {, if } \boldsymbol{P}: \boldsymbol{A} \geq 0 \\ (1+\boldsymbol{P}: \boldsymbol{A})^{n_{P}}\left(1-\frac{\mathrm{C}_{P}}{\mathrm{C}_{S D}+\mathrm{C}_{P}} \frac{\mathrm{S}_{D}}{\mathrm{~S}_{\text {sat }}}\right), \text { otherwise. }\end{array}\right.$

The evolution of $\boldsymbol{S}_{\mathrm{L}}$ results from the interaction between microbands generated by newly activated slip planes after a strain-path change and the preformed dislocation walls.

$\dot{\boldsymbol{S}}_{\mathrm{L}}=-C_{\mathrm{SL}}\left(\frac{\left|\boldsymbol{S}_{\mathrm{L}}\right|}{S_{\text {sat }}}\right)^{n_{\mathrm{L}}} \boldsymbol{S}_{\mathrm{L}} \dot{\bar{\varepsilon}}$

where $C_{\mathrm{SL}}$ characterizes the rate of disorganization of the preformed dislocation structures.

The yield stress is given by

$\sigma_{\mathrm{Y}}=Y_{0}+R+m S$,

where $Y_{0}$ is the initial yield stress, $m$ is a material parameter and $S$ is the norm of $\boldsymbol{S}$, i.e.

$S=|\boldsymbol{S}|=\sqrt{\left|\boldsymbol{S}_{\mathrm{L}}\right|^{2}+S_{\mathrm{D}}^{2}}$.

The model involves 13 material parameters, namely $Y_{0}, X_{0}, S_{\mathrm{sat}}, R_{\mathrm{sat}}, C_{\mathrm{P}}, C_{\mathrm{SL}}, C_{\mathrm{SD}}, C_{\mathrm{X}}, C_{\mathrm{R}}, n_{\mathrm{P}}, n_{\mathrm{L}}, m$ and $q$

\section{The Yld2000-2d Yield Function}

The Yld2000-2d plane stress yield function introduces the plastic anisotropy by using two linear transformations on the Cauchy stress tensor (Barlat et al., 2003). It is expressed in terms of the deviatoric stress components (Barlat et al., 2007) as

$\phi=\phi^{\prime}\left(\tilde{\boldsymbol{S}}^{\prime}\right)+\phi^{\prime \prime}\left(\tilde{\boldsymbol{S}}^{\prime \prime}\right)=2 \bar{\sigma}_{\mathrm{Y}}^{a}$

where $\phi^{\prime}$ and $\phi^{\prime \prime}$ are two isotropic functions defined by

$\phi^{\prime}\left(\tilde{\boldsymbol{S}}^{\prime}\right)=\left|\tilde{S}_{1}^{\prime}-\tilde{S}_{2}^{\prime}\right|^{a}$

$\phi^{\prime \prime}\left(\tilde{\boldsymbol{S}}^{\prime \prime}\right)=\left|2 \tilde{S}_{2}^{\prime \prime}+\tilde{S}_{1}^{\prime \prime}\right|^{a}+\left|2 \tilde{S}_{1}^{\prime \prime}+\tilde{S}_{2}^{\prime \prime}\right|^{a}$

while $\tilde{\boldsymbol{S}}^{\prime}$ and $\tilde{\boldsymbol{S}}^{\prime \prime}$ are linear transformations of the effective stress tensor $\boldsymbol{s}$, which is defined as difference between the deviatoric part $\boldsymbol{\sigma}^{\prime}$ of the Cauchy stress and the back stress $\boldsymbol{X}$, i.e.

$\boldsymbol{s}=\boldsymbol{\sigma}^{\prime}-\boldsymbol{X}$. 
Hence

$\tilde{\boldsymbol{S}}^{\prime}=\boldsymbol{C}^{\prime} \boldsymbol{s}, \quad \tilde{\boldsymbol{S}}^{\prime \prime}=\boldsymbol{C}^{\prime \prime} \boldsymbol{s}$,

where $\boldsymbol{C}^{\prime}$ and $\boldsymbol{C}^{\prime \prime}$ represent the linear transformations of the effective stress tensor $\mathbf{s}$ and contain the material anisotropy coefficients, which are computed using as input the experimental values of the stresses and anisotropy factors in tension along three directions and the balanced biaxial flow stress as well as the balanced biaxial anisotropy coefficient.

\section{RESULTS AND DISCUSSION}

\section{Materials Characterization}

Two metal sheets of deep-drawing quality were considered in our study, namely an aluminum-killed and titanium-treated mild steel (DC06 steel) and a bake-hardened steel (BH steel). Table 1 presents the corresponding values of the yield stress and anisotropy factor $r$ for three orientations of the uniaxial tensile direction with respect to the rolling direction, ultimate tensile strengths, maximum uniform elongations, as well as the balanced biaxial yield stress $\sigma_{\mathrm{b}}$ and the balanced biaxial anisotropy coefficient $r_{\mathrm{b} .}$.

TABLE 1. The material plastic properties

\begin{tabular}{lccccccc}
\hline Material & Orientation & $\begin{array}{c}\sigma_{\mathbf{Y}} \\
{[\mathbf{M P a}]}\end{array}$ & $\begin{array}{c}\sigma_{\mathbf{u t s}} \\
{[\mathbf{M P a}]}\end{array}$ & $\begin{array}{c}\text { Uniform } \\
\text { elongation }\end{array}$ & $\begin{array}{c}\text { Anisotropy } \\
\text { factor } \boldsymbol{r}\end{array}$ & $\boldsymbol{\sigma}_{\mathbf{b}}$ & $\mathbf{r}_{\mathbf{b}}$ \\
\hline DC06 steel & $0^{\mathbf{o}}$ & 136 & 374 & $29 \%$ & 2.6 & 142.2 & 0.8 \\
& $45^{\circ}$ & 134 & 369 & $27 \%$ & 2.1 & & \\
\multirow{3}{*}{ BH steel } & $90^{\circ}$ & 136 & 365 & $28 \%$ & 3.2 & & \\
& $0^{\circ}$ & $210 / 208$ & 410 & $23 \%$ & 2.2 & 216.56 & 0.869 \\
& $45^{\circ}$ & $224 / 218$ & 417 & $21 \%$ & 1.5 & & \\
\hline
\end{tabular}

Table 2 contains the material parameters involved in the microstructural hardening model.

TABLE 2. The material parameters of the microstructural model

\begin{tabular}{|c|c|c|c|c|c|c|c|c|c|c|c|c|c|}
\hline Parameters & $Y_{0}$ & $C_{\mathrm{R}}$ & $R_{\text {sat }}$ & $C_{\mathrm{X}}$ & $X_{0}$ & $C_{\mathrm{SD}}$ & $C_{\mathrm{SL}}$ & $S_{\text {sat }}$ & $\mathbf{n}_{\mathbf{L}}$ & $\mathbf{n}_{\mathbf{p}}$ & $q$ & $m$ & $C_{\mathrm{P}}$ \\
\hline DC06 steel & 121.1 & 31.9 & 90.0 & 446 & 15.9 & 4 & 1.86 & 231.1 & 0 & 27.9 & 1.5 & 0.445 & 5.5 \\
\hline BH steel & 202.93 & 37.2 & 73.45 & 160.15 & 2.33 & 4.93 & 5.575 & 229.8 & 0.25 & 24.05 & 1.855 & 0.297 & 3.5 \\
\hline
\end{tabular}

Table 3 shows the values of the eight parameters of the Yld00-2d yield function, as obtained by a numerical identification from the experimental data: $r_{0}, r_{45}, r_{90}, r_{\mathrm{b}}, \sigma_{0}, \sigma_{45}, \sigma_{90}, \sigma_{\mathrm{b}}$.

TABLE 3. The coefficients of the Yld00-2d yield function

\begin{tabular}{lccccccccc}
\hline Material & $\boldsymbol{a}$ & $\boldsymbol{\alpha}_{\mathbf{1}}$ & $\boldsymbol{\alpha}_{\mathbf{2}}$ & $\boldsymbol{\alpha}_{\mathbf{3}}$ & $\boldsymbol{\alpha}_{\mathbf{4}}$ & $\boldsymbol{\alpha}_{\mathbf{5}}$ & $\boldsymbol{\alpha}_{\mathbf{6}}$ & $\boldsymbol{\alpha}_{7}$ & $\boldsymbol{\alpha}_{\mathbf{8}}$ \\
\hline DC06 steel & 6 & 1.055 & 1.09 & 1.00 & 0.9 & 0.937 & 1.056 & 1.069 & 0.926 \\
BH steel & 6 & 1.072 & 1.039 & 1.027 & 0.916 & 0.943 & 1.0423 & 0.982 & 0.837 \\
\hline
\end{tabular}

\section{Strain and Stress Based Forming Limits: Computation and Discussion}

In order to achieve the best agreement with the experimental data, the initial value of the MK geometrical defect was individually selected for each material, namely 0.996 for DC06 steel and 0.994 for BH steel. The shape of yield surface is described by the YLD00-2d yield function and the strain hardening is defined by the microstructural hardening model. Figure 1 shows the experimental and theoretical forming limits for DC06 steel under proportional (acronym LSP) and non proportional loadings involving two strain path changes, maintaining constant the first and second stress ratio (acronym $\alpha$ ) and varying the amount of prestrain under uniaxial tension (acronym UT), biaxial stretching (acronym BS) and plane strain (acronym PS), respectively. Figure 2 shows the experimental and 
theoretical FLDs for BH steel sheet determined under linear and non-proportional loading using a sequence of two linear strain paths, keeping constant the preliminary strain/stress ratio and the amount of prestrain and varying the subsequent stress ratio. Specifically, it was considered prestrain at several values under UT, BS and PS followed by different strain paths (acronym X) between UT and BS. A good correlation between experimental data and the predicted results for linear and complex strain paths is observed for both of the selected materials. It may be reasonable assumed that this remarkable accuracy in the FLD prediction results from the relevance of the applied constitutive models. Concerning the complex strain paths, it is observed that UT prestrain along RD shifts the whole FLD to the left, raising the strain limits in biaxial tension region and consequently increasing the slope of right-hand side of the FLD. If the first stage of strain is under BS, the subsequent FLD shifts to the right and down with respect to the original FLD, while under PS prestrain the slopes of right-hand side and left-hand side of FLD increase. Moreover, the strong effect of the amount of prestrain in the predicted forming limits under complex strain paths is clearly showed in Figure 1. It is noticed a decrease of forming limits for small values of the prestrain followed by an increase of forming limits as the prestrains increase. Such effect of decrease of forming limits is much pronounced when the second strain path is between UT and PS and it becomes softer (for BS prestrains) and even disappears (for UT prestrains) as the strain ratio characterizing the second strain path increases in the range PS-BS.

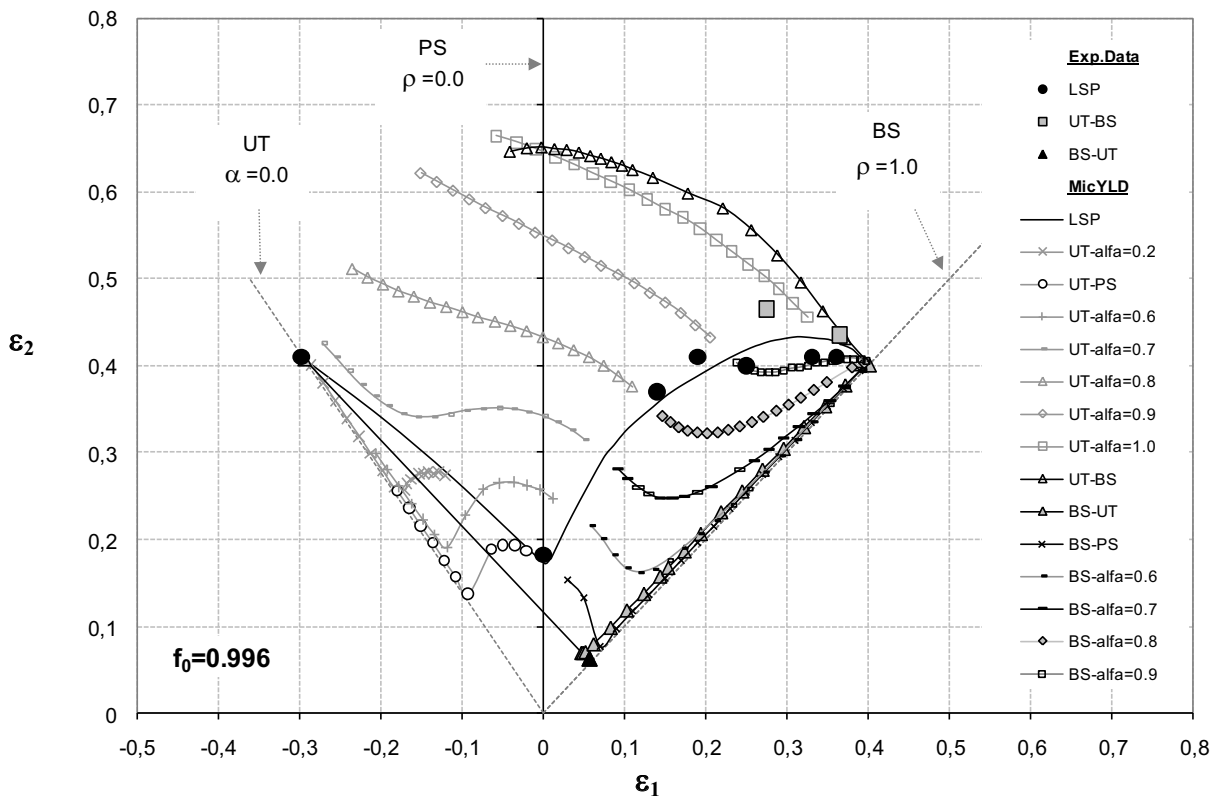

FIGURE1. Forming Limit Diagram under linear and complex strain paths for DC06 steel.

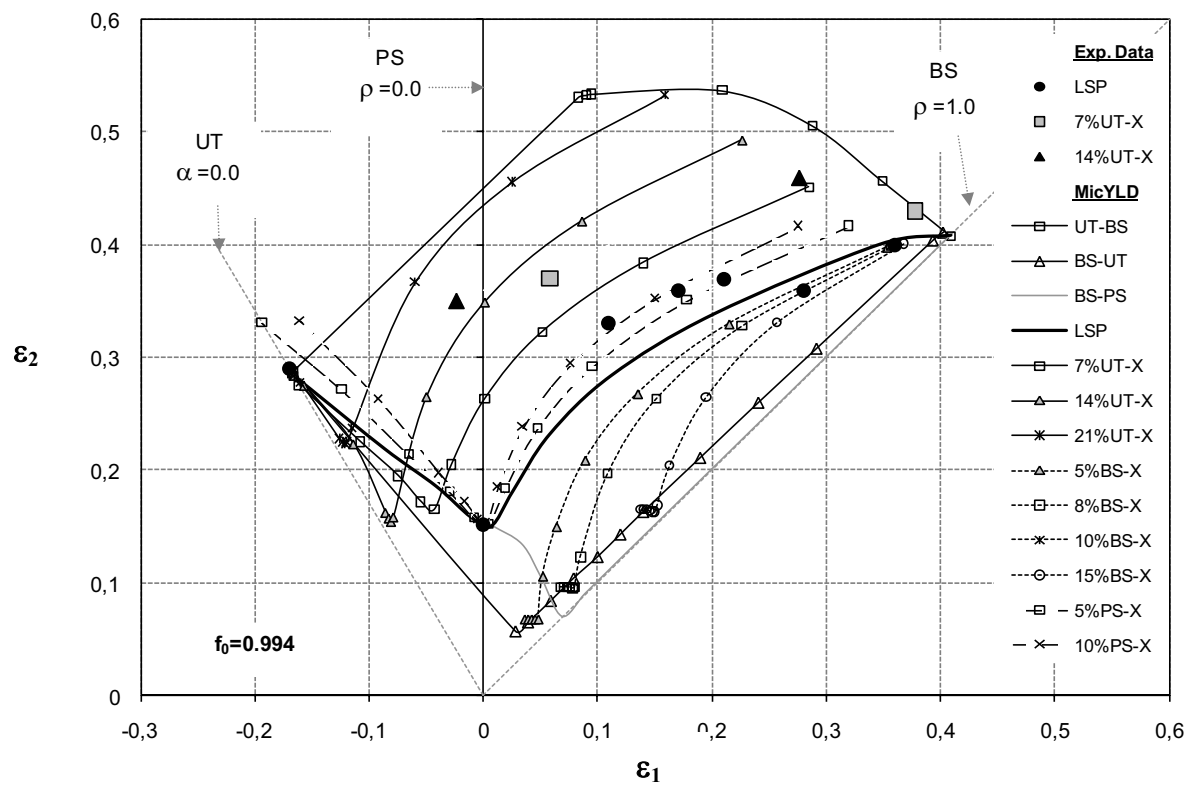

FIGURE2. Forming Limit Diagram under linear and complex strain paths for BH steel. 
Figure 3 shows the stress-based forming limits for DC06 steel under linear and complex strain paths. The effect of the amount of prestrain in the predicted stress-based forming limits is clearly observed. Figure 4 shows the stressbased forming limits for BH steel under linear and complex strain paths involving prestrain at different values under UT, BS and PS , respectively, following by different strain paths. All the results illustrate a well defined effect of the strain path change on the predicted stress-based forming limits. This dependence of the stress-based forming limits in the strain path change is due to the strain path induced anisotropy that is taken into account by the microstructural hardening model based on the dislocation structure. It is very interesting to observe that in general the stress-based forming limits under almost all selected strain path changes are higher than the FLSD under linear strain paths. Moreover excepting few cases for small UT prestrains values the stress-based forming limits increase with the increase of prestrain value for all assumed prestrains.

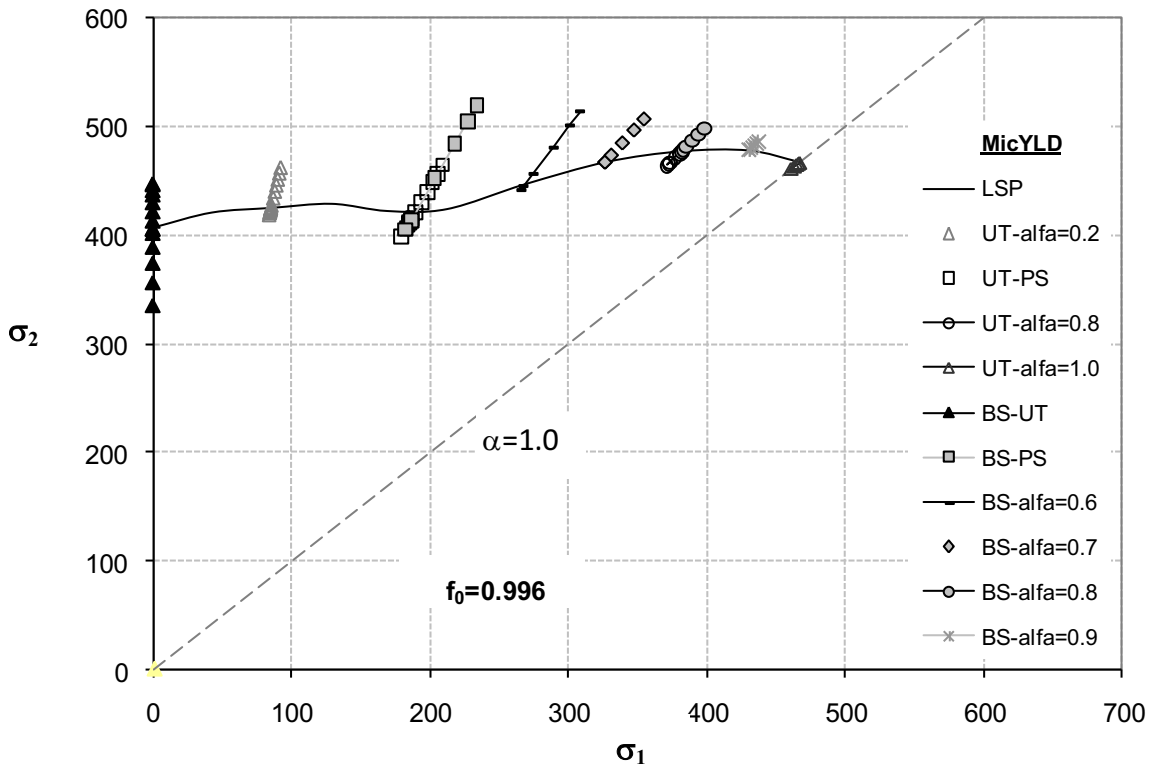

FIGURE3. Forming Limit Stress Limits under linear and complex strain paths for DC06 steel.

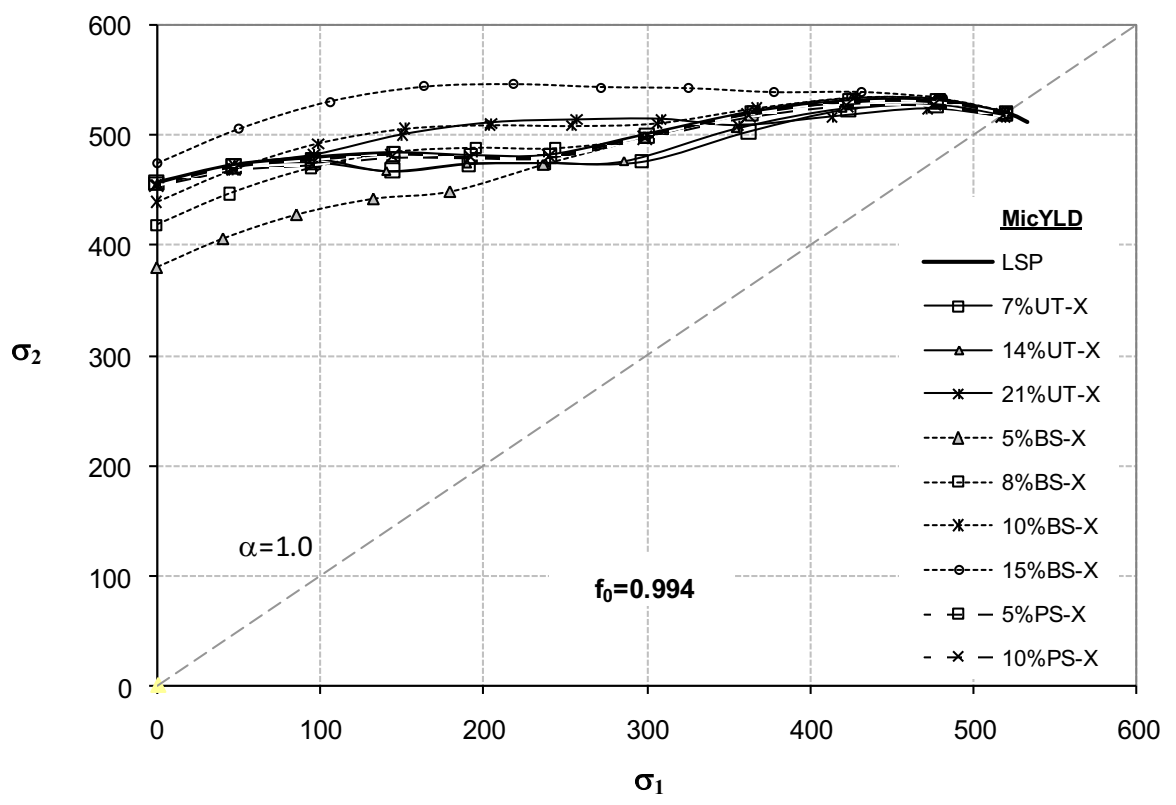

FIGURE4. Forming Limit Stress Diagram under linear and complex strain paths for BH steel. 
Figures 1 and 2 show that in both of studied cases the lowest curve on the FLD is the one obtained for BS-UT and the uppermost curve is the one obtained for UT-BS. It has been found an increase of formability under UT-BS until 25\% for DC06 steel and 13\% for BH steel, as well as a loss of formability under BS-UT until 33,8\% for DC06 steel and $23,3 \%$ for $\mathrm{BH}$ steel, although these two deformation histories have the same value of the parameter introduced by Schmitt et al. [7] to characterize the strain-path changes (namely 0.269). Actually, the earlier appearance of plastic instability under the sequence BS-UT may be attributed to the fact that the second path is the uniaxial tension, which is more sensitive to strain localization. For UT-BS, the balanced biaxial stress state leads to a very stable strain path. Therefore, the occurrence of plastic flow localization is controlled primarily by the occurrence of a geometrical instability (necking in tension). This geometrical instability is captured in the MK model through its necking criterion. Figure 5 shows the evolution of the MK necking criterion for different strain paths for DC06 steel. As expected, the MK necking criterion is firstly attained for BS-UT and lastly for UT-BS, fact which explains the forming limits results. Figure 5 also shows that the choice of the MK necking criterion is practically irrelevant for the computed FLDs, as the strain-rate in the groove increases very rapidly after this condition is attained, because of the low current hardening rate.

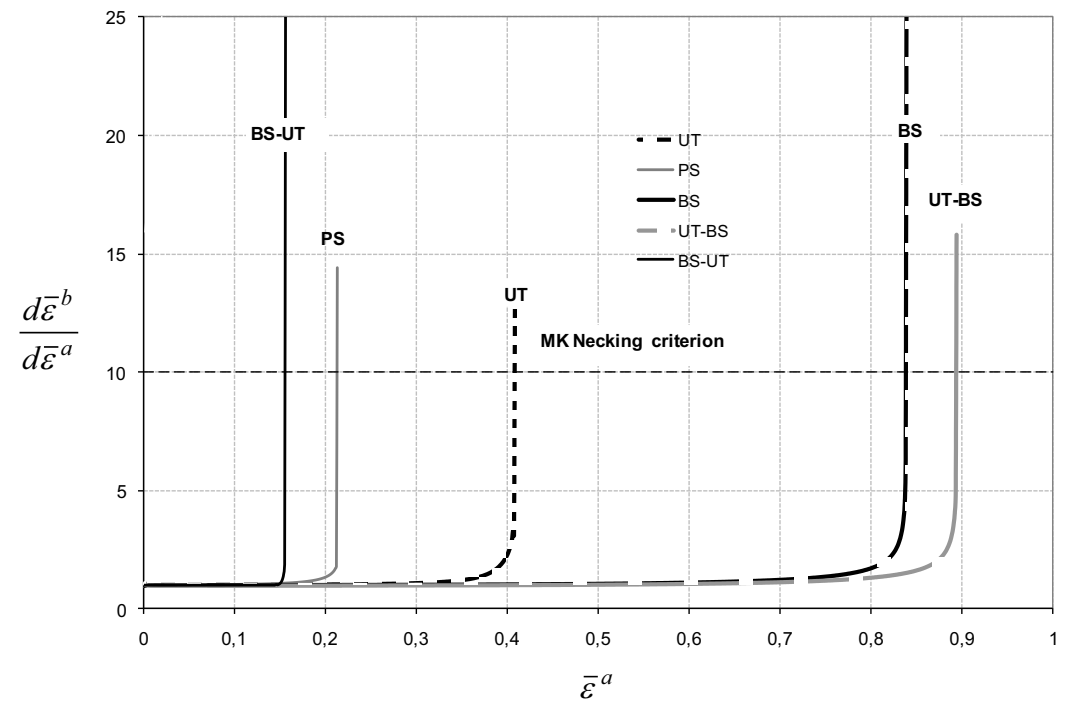

FIGURE5. Evolution of the MK necking criterion for different strain paths for DC06 steel

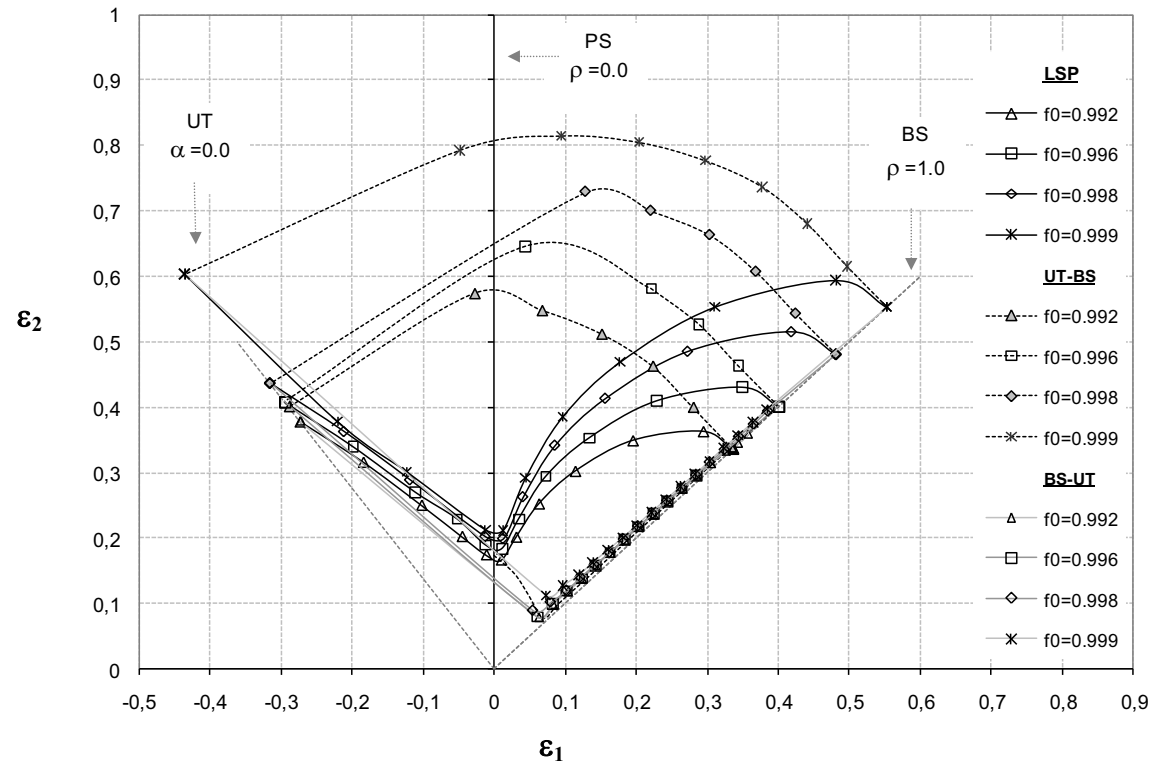

FIGURE6. The Influence of the initial value of MK geometrical defect in FLD under linear and complex strain paths for DC06 steel 
Figure 6 shows the significant influence of the initial value of MK geometrical defect in the predicted FLD under linear and complex strain paths for DC06 steel. A slight increase of initial value of the MK geometrical defect allows to a considerable increase of the predicted forming limits under UT-BS and in the right-hand side of the FLD under LSP. Such increase of the forming limits is much smaller in the left-hand side of the FLD under LSP and under BS-UT.

\section{CONCLUSIONS}

An advanced model for FLD prediction based on MK theory has been used to predict the strain and stress-based forming limits for DC06 steel sheet and BH steel sheet under linear and complex strain paths. A very good agreement between experiments and theoretical results for the selected materials has been found. The great accuracy on the prediction of the forming limits is due to the ability of the microstructural hardening model of Teodosiu and $\mathrm{Hu}$, which accounts for the anisotropic work-hardening induced by the microstructural evolution at large strains and of the Yld2000-2d yield function to describe in great detail the material behavior. The plastic instability prediction was performed for several complex strain paths. The strain path change clearly influences the plastic flow localization. It is notable the significant decrease/increase of formability that occurs under strain histories involving a biaxial stretching prestrain followed by uniaxial tension and a uniaxial tensile prestrain followed by biaxial stretching even if these two sequences have the same value of the Schmitt factor to characterize the strain path change. The occurrence of plastic flow localization is controlled primarily by the occurrence of a geometrical instability (necking in tension). This geometrical instability is captured in the MK model through its necking criterion. Theoretically, the occurrence of plastic flow localization is mainly connected with the accomplishment of the $\mathrm{M}-\mathrm{K}$ necking criterion, which is strictly dependent on the assumed strain path. Taking into account the strain path induced anisotropy through the microstructural hardening model it was proved that the stress-based forming limits are also considerable affected by the strain path changes. In addition it is noticed that the stress-based forming limit concept is not efficient to evaluate the sheet metal formability, since under specific complex loadings (i.e BSUT) a significant increase of the stress-based forming limits while a considerable decrease of the strain-based forming limits was observed.

\section{ACKNOWLEDGMENTS}

Especial thanks are expressed to Prof. Cristian Teodosiu for all support concerning the microstructural hardening model. The authors thank Laboratory of the Mechanical and Thermodynamic Properties of Materials (LPMTM, U. Paris Nord, France) for providing the material parameters of microstructural model.

\section{REFERENCES}

[1] Marciniak, Z., Kuczynski, K., Pokora, T., "Influence of The Plastic Properties of A Material On The Forming Limit Diagram For Sheet Metal In Tension", Int. J. Mech. Sci., 15, 789-805 (1973).

[2] Barlat, F., Brem, J. C., Yoon, J. W., Chung, K., Dick, R. E., Lege, D. J., Pourboghrat, F., Choi, S. -H. Chu E., "Plane stress yield function for aluminum alloy sheets-part 1: theory" Int. J. Plasticity, 19, 1297-1319 (2003).

[3] Teodosiu C., Hu Z., "Microstructure in the continuum modelling of plastic anisotropy" In: Roskilde. Danemark (Eds.). Proc. Of the $19^{\text {th }}$ Riso International Symposium on Modelling of Structures and Mechanics of Materials from Microscale to Product , 149-168 (1998).

[4] M.C. Butuc, J.J. Gracio and A. Barata da Rocha, "An experimental and theoretical analysis on the application of stressbased forming limit criterion”, Int. J. Mech. Sci., 48 (4), 414-429 (2006)

[5] Yoshida, Kengo, Suzuki, Noriyuki "Forming limit stresses predicted by phenomenological plasticity theories with anisotropic work-hardening behavior" Int. J. Plasticity 24, 118-139 (2008)

[6] M.C. Butuc , F. Barlat, J.J. Grácio, A. Barata da Rocha, "A new model for FLD prediction based on advanced constitutive equations", Int. J. of Mat. Forming, DOI 10.1007/s12289-009-0667-6 (2009)

[7] Schmitt, J.-H., Shen E.L., Raphanel J.L., 1994, A parameter for measuring the magnitude of a change of strain path: Validation and comparison with experiments on low carbon steel. Int. J. Plasticity 10, 535. 